\title{
Le corps, le rythme et l'esthétique sociale chez André Leroi-Gourhan
}

\section{Alexandra Bidet}

\section{(2) OpenEdition}

Édition électronique

URL : https://journals.openedition.org/tc/2132

DOI : $10.4000 /$ tc. 2132

ISSN : 1952-420X

Éditeur

Éditions de l'EHESS

\section{Édition imprimée}

Date de publication : 12 décembre 2007

Pagination : 15-38

ISSN : 0248-6016

\section{Référence électronique}

Alexandra Bidet, « Le corps, le rythme et l'esthétique sociale chez André Leroi-Gourhan », Techniques \&

Culture [En ligne], 48-49 | 2007, mis en ligne le 20 juin 2010, consulté le 29 septembre 2022. URL :

http://journals.openedition.org/tc/2132 ; DOI : https://doi.org/10.4000/tc.2132 


\title{
Alexandra Bidet*
}

\section{Le corps, le rythme et l'esthétique sociale chez André Leroi-Gourhan}

\begin{abstract}
L'ouvre d'André Leroi-Gourhan est traversée par une anthropologie du rythme. Celle-ci ne part pas d'une socialité constituée, de rythmes dits "sociaux", mais inscrit au contraire l'analyse de la rythmicité dans une approche de l'homme comme être vivant, comme totalité indivise. Elle pose en des termes renouvelés le problème classique du groupement des hommes et des liens entre lindividu et son milieu. Avec la question de la genèse de la socialité, c'est le lien entre corporéité et socialité, entre affect et symbole, qui est ici au cour d'une approche du rythme comme « insertion dans l'existence».
\end{abstract}

Corps, rythmicité, activité technique, insertion sociale, symbolisme.

"L'homme est une création du désir, non pas une création du besoin. " G. Bachelard, La Psychanalyse du feu, cité par A. Leroi-Gourhan (I982 : I80).

"On connaît mieux les échanges de prestige que les échanges quotidiens, les prestations rituelles que les services banaux, la circulation des monnaies dotales que celle des légumes, beaucoup mieux la pensée des sociétés que leur corps [...]. Alors que Durkheim et Mauss ont luxueusement défendu le "fait social total", ils ont supposé linfrastructure techno-économique connue» (Leroi-Gourhan I964 : 210).

Le rythme serait-il rétif à toute forme de pensée? On a pu souligner la polysémie de la notion de rythme, les apories des démarches qui visent à lui assigner une origine, ou encore l'existence d'un "panrythmisme " spontané prompt à déceler du rythme en toutes choses (Sauvanet \& Wunenburger I996). Dans l'anthropologie, l'archéologie et l'ethnologie préhistorique d'André Leroi-Gourhan, une place majeure est accordée à la rythmicité et

* Chargée de recherche au CNRS, Centre Maurice Halbwachs CNRS-EHESS-ENS. 
aux rythmes sans qu'il s'agisse de saisir le rythme comme l'essence même de l'homme, ou de le dissoudre dans la pluralité de ses manifestations sensibles. Son ouvre, dont on a souvent noté la valeur proprement philosophique, dessine bien au contraire une véritable anthropologie du rythme -compris comme « insertion affective » ou « insertion dans l'existence » (Leroi-Gourhan I965a : 8I-82, IO2). Incarnant la continuité de "l'ordre physiologique technique- social»(Ibid.: 87), il émarge alors d'emblée aux phénomènes «sociaux».

À linitiative d'un profond bouleversement du regard porté par les recherches préhistoriques sur lart des premiers anthropiens et sur le processus d'hominisation lui-même, cette œuvre est pourtant demeurée longtemps peu fréquentée des sociologues ${ }^{1}$. S’il est commun de rappeler qu'André Leroi-Gourhan fut lélève de Marcel Mauss, rares sont les investigations dépassant l'apport strictement ethnologique et technologique de l'œuvre, principalement perçue comme une vaste entreprise de recensement et de classification des techniques, appréhendées à partir du geste humain lui-même, sur un mode emprunté aux sciences naturelles, et plus particulièrement à la taxinomie. Or sa réévaluation de la notion de rythme, attentive aux aspects empiriques et corporels de l'existence humaine, place la réflexion de Leroi-Gourhan au coeur des préoccupations sociologiques, de plus en plus soucieuses du lien entre corporéité et socialité.

Menant dans Le Geste et la Parole, dont le second tome sintitule La Mémoire et les Rythmes, une réflexion d'ensemble sur son ouvre, André Leroi-Gourhan ne se donne jamais pour objet de développer une pensée systématique du rythme. De la "mémoire sociale" aux «symboles de la société ", la notion de rythme traverse néanmoins l'analyse de la genèse de l'esthétique et du social, qui fait suite à celle de la technicité et du langage, étudiée dans le premier tome ${ }^{2}$. C'est à une lecture en biais qu'il faut alors procéder pour saisir une conceptualisation qui ne se départit jamais de considérations empiriques. Dans la lignée du projet maussien d’analyse des

\footnotetext{
${ }^{1}$ Cette $œ u v r e$, et plus généralement la tradition française d'anthropologie technique (notamment Marcel Mauss et Gilbert Simondon), constituent depuis une dizaine d'années l'une des principales références du renouveau des théories sociologiques de l'action et des approches de l'activité (Schwartz 1988, 2001 ; Latour 1991 ; Breviglieri 1993 ; Thévenot 1994 ; Dodier 1995 ; Clot 1995 ; Kaufmann 1997, 2001 ; Bidet 2001).

2 Dans ce volume, des titres de chapitres ou sections font explicitement référence à la question du rythme : «Les fondements corporels des valeurs et des rythmes », « Les rythmes », «Le rythme figuré » $(1964$ : 95, 135, 216)
} 
«techniques du corps» et de l'«homme total» (Karsenti $1998: 238)^{3}$, son anthropologie du rythme ne part pas d'une socialité constituée, celle des rythmes dits "sociaux", mais reconduit l'analyse de la rythmicité à une analyse de l'homme comme être vivant, comme totalité biologique indivise. Elle appréhende les phénomènes rythmiques de façon continue depuis l'espace du vital et du physiologique, à travers ce qui constitue en propre le vivant: l'activité par laquelle il ne cesse de se produire dans sa relation problématique à son milieu de vie.

La référence au vital ne désigne pas ici une essence de l'homme, appelée à sactualiser, mais au contraire la façon propre à l'homme de problématiser son rapport au milieu, done de s'inventer. Ce style spécifiquement humain émane tout entier pour André Leroi-Gourhan de la condition naturelle que son corps fait à l'homme, par sa structure morphologique et ses possibilités dynamiques. À l'opposé de tout naturalisme, elle "voit émerger le vital comme spécifiquement humain, et donc social» (Karsenti 1998: 238). L'attention à la dynamique du vital ouvre alors, à travers la question du rythme, sur une genèse du social lui-même, posant en des termes renouvelés la question de "linsertion dans l'existence" et du "groupement des hommes", ce "tissu de relations entre lindividu et le groupe " (LeroiGourhan I965a : II, 80, 89). Le statut accordé aux phénomènes rythmiques vient interroger les catégories ordinaires de lanthropologie et de la sociologie. Mais sur quelle caractérisation de la rythmicité un tel renversement repose-t-il ?

\section{De la corporéité aux rythmes}

L'attention portée à la rythmicité caractérise une anthropologie ancrée dans l’analyse du corps humain. Considéré par André Leroi-Gourhan comme analytiquement premier pour toute étude de l'activité humaine, il l'est d'abord du point de vue de la genèse de l'homme comme espèce, à laquelle l'auteur

\footnotetext{
3 B. Karsenti souligne combien la conceptualisation développée par A. Leroi-Gourhan ne peut se comprendre hors de «l'inversion méthodologique accomplie dans le cadre inaugural de la sociologie de M. Mauss » et son parti pris d'adopter pour centre «l'espace corporel», « l'élément du physiologique» et les «montages physiopsycho-sociologiques » qui trament l'existence concrète de l'homme (1998 : 228). Par «homme total ", Mauss entend en effet le caractère indissociable chez l'homme du physiologique, du psychologique et du social. Par l'expression de "techniques du corps ", il désigne le corps comme le premier et le plus naturel instrument de l'homme, un instrument dont les usages ne sont toutefois pas naturels mais bien l'objet d'un apprentissage.
} 
consacra la majeure partie de ses travaux en paléontologie humaine. Seule l'originalité morphologique et fonctionnelle du corps humain, qui spécifie le type hominien sur l'échelle des espèces, autorise à ses yeux une compréhension du style original des rapports que l'homme entretient quotidiennement avec son milieu de vie.

Dans les années 1960, la découverte de lindustrie des australopithèques -jusqualors regardés comme anthropoïdes pour leur très faible volume crânien- a introduit une révolution décisive dans le domaine de la paléontologie. Elle attestait l'antériorité des premières formes de technicité sur le développement cérébral lui-même, c'est-à-dire l'antériorité de larchitecture générale du corps humain - caractérisée par la station verticale et la libération de la main de la locomotion - sur la commande nerveuse et l'expansion du cortex cérébral. Dans l'histoire du couple main-cerveau, la libération de la main n’était plus le produit de l'apparition de la pensée, mais son moteur même : "la main qui libère la parole, c'est exactement ce à quoi aboutit la paléontologie", même si "nous étions préparés à tout admettre sauf d'avoir commencé par les pieds " (Leroi-Gourhan I964:33, 40, 7I, 97). Dans le développement cérébral, la main et les outils qu'elle génère sont en constante interaction avec la face et le langage qui peu à peu s'en libèrent. Activité motrice et activité verbale sont indissociables.

"Un des résultats de l'étude simultanée de l'homme sous les angles de la biologie et de l'ethnologie est de montrer le caractère inséparable de l'activité motrice (dont la main est l'agent le plus parfait) et de lactivité verbale. Il n'existe pas deux faits typiquement humains dont l'un serait la technique et l'autre le langage, mais un seul phénomène mental, fondé neurologiquement sur des territoires connexes, et exprimé conjointement par le corps et par les sons. La prodigieuse accélération du progrès à partir du déverrouillage des territoires préfrontaux est liée à la fois au déversement du raisonnement dans les opérations techniques et à linféodation de la main au langage dans le symbolisme graphique qui aboutit à l'écriture » (1965a : 259-260).

L'originalité radicale du type hominien s'ancre ainsi dans la spécificité morphologique et fonctionnelle du corps humain. Elle se marque par labsence de toute spécialisation fonctionnelle: cette indétermination native contraste radicalement avec l'adaptation efficace et étroite des formes animales aux caractéristiques déterminées d'un biotope. Susceptible d'une gamme indéfiniment variée de modes d'interaction avec son milieu, l'homme n’a pas connu d'évolution spécialisante; il demeure marqué par la généralité et la polyvalence - perceptibles dans la seule forme du pied ou de la main. Or, le caractère indifférencié de cet héritage naturel, cette polyvalence 
posturale, gestuelle et fonctionnelle, ont pour inévitable contrepartie une extrême faiblesse native, un dénuement inhérent à l'absence d'une adaptation préformée à un cadre fonctionnel d'origine naturelle.

Ce double principe de plasticité et d'incomplétude est essentiel. Là réside pour André Leroi-Gourhan le caractère foncièrement problématique du rapport de l'homme à son milieu de vie : il n’indique pas des besoins à satisfaire, ou des fins présidant à son adaptation, mais une puissance d'agir, trouvant sa propre fin dans sa capacité d'invention infinie de problèmes et de médiations. La notion de problème ne recouvre donc pas un ensemble donné de prémisses et de solutions, mais désigne lincertitude propre au rapport que l'activité humaine entretient avec son milieu - rapport dont la nature et les produits sont indéterminés ${ }^{4}$. Rites, règles et outils n’émergent donc pas tels des pouvoirs préformés, contenus dans la structure corporelle de l'homme, mais comme des inventions face au défi biologique que constitue pour lui l'absence de prise "native " sur son milieu5. Son inventivité relève alors d'une dynamique vitale, d'une exigence d'appartenance, mais radicalement affranchie du cadre zoologique: il engage linvention de déterminations proprement humaines; il est création plus que conservation: "l'animal répète là où lêtre humain, parce qu’il n’a pas en lui de schémas de comportement innés, est obligé d'inventer ».

«Station verticale, polyvalence des membres antérieurs, conservation des traits infantiles, extrême lenteur de croissance, et donc aussi dépendance prolongée par rapport aux parents sont des aspects corrélatifs qui définissent les grandes lignes d'une insertion dans le monde d'une originalité telle qu'elle introduit à une différence d'un autre ordre que celles qui distinguent les espèces. Défi auquel il conviendra de répondre par la constitution d'un réseau propre de moyens, et plus encore de normes et de régulations ou de disparaitre " (Tinland $1977: 76$ ).

L'attention à la rythmicité découle directement de cette focalisation sur le corps et la motricité humaine. Elle permet en effet de saisir concrètement, au ras de l'ordre physiologique, la question centrale pour André Leroi-Gourhan de «linsertion dans l'existence». L'application des premières percussions

\footnotetext{
${ }^{4}$ Comme chez G. Simondon, et en une même filiation bergsonienne, il s'agit moins de penser une «morphologie» qu'une "énergétique humaine ", moins du social substantifié que la dynamique même de la formation et de la transformation des groupes humains (Simondon 1989). Ce qui fonde cette pensée de la genèse est une attention fondamentale au caractère problématique du vivant - soit à la catégorie de problème, qui remplace à partir de H. Bergson celle du « besoin » dans la pensée du vivant (Douçot 2008).

5 Elle anime «l'axiomatique ouverte des problèmes vitaux », selon la formulation de G. Simondon (1989) : le vivant, défini comme «être problématique », se caractérise par sa capacité d' « individuation permanente ».
} 
rythmiques, puis l'apparition des premières représentations rythmiques, sous la forme de "fragments d'os en pierre marqués d'incisions régulièrement espacées", marquent bien à ses yeux "l'entrée dans l'humanité des Australanthropes "6 (1965a: 135 ). Du "rythme technique" aux "rythmes figuratifs ", le rapport au milieu voit ici la genèse continuée de médiations :

"Au piétinement qui constitue le cadre rythmique de la marche, s'ajoute chez l'homme
lanimation rythmique du bras; alors que le premier régit l'intégration spatio-temporelle
et se trouve à la source de l'animation dans le domaine social, le mouvement rythmique du
bras ouvre une autre issue, celle d'une intégration de lindividu dans un dispositif créateur
non plus d'espace et de temps mais de formes. La rythmicité du pas a finalement abouti
au kilomètre et à l'heure, la rythmicité manuelle a conduit vers la capture et
limmobilisation des volumes, source d'une réanimation purement humaine. Du rythme
musical, tout de temps et de mesures, au rythme du marteau ou de la houe, tout de
procréation de formes, immédiates ou différées, la distance est considérable puisque l'un
est générateur d'un comportement qui trace symboliquement la séparation du monde
naturel et de l'espace humanisé alors que le second transforme matériellement la nature
sauvage en instruments de l'humanisation. L'une et l'autre sont strictement
complémentaires " (ibid.: 136 ).

La primauté accordée au corps comme motricité fait done ici tout le statut de la rythmicité. C'est par la motricité que s'opère le passage de la corporéité à la rythmicité. Ce faisant, André Leroi-Gourhan nous introduit aussi d'emblée à la dimension de l'affect.

\section{Du rythme comme "insertion affective»: rythme et valeurs}

Ainsi posé depuis la motricité la plus élémentaire, le problème de l'« insertion dans l'existence» peut alors s'énoncer en termes de rythmicité, comme une "insertion affective". Dire que "l'équilibre rythmique " est senti dans " toute l'épaisseur de la vie sensitive", dès "la sensibilité viscérale et musculaire profonde " signifie en effet qu’il dépasse le seul registre de la sensation pour désigner un état d'ensemble de la corporéité, soit l'état affectif, entendu comme ce qui touche et pousse à agir, l'élément déterminant de toute mise en situation (Leroi-Gourhan 1965a : 99). André Leroi-Gourhan se plaît ainsi à rappeler que «linsertion cosmique " part de l'estomac et de la "satisfaction digestive ", tout comme "la désinsertion cosmique débute au niveau du tube digestif " (Ibid.: IO2). De même, la référence à la sensation du "poids du

\footnotetext{
${ }^{6}$ A. Leroi-Gourhan regroupe dans la catégorie des australanthropes les premiers anthropiens. Identifiés en Afrique entre la fin du tertiaire et le début du quaternaire, ils sont les premiers à présenter la forme qu'il caractérise comme proprement hominienne.
} 
corps" et celle de "l'équilibre spatial ", comme aux mouvements les plus viscéraux du comportement nutritif et de l'affectivité physique respectivement fondés sur les rythmes viscéraux et sur la perception du jeu musculaire (Ibid. : IO3) - noue ici "insertion dans l'existence", rythmes et affectivité : la médiation s'opère dans l'élément de l'affect, la rythmicité produisant cette " insertion affective ".

Mais le rythme a deux visages chez André Leroi-Gourhan : il désigne autant les chaînes rythmiques propres à nos gestes "stéréotypés ", que toute soustraction à cette «ambiance rythmique ». D'un côté, «la répétition assure l'équilibre normal du sujet dans le milieu social et son propre confort psychique à lintérieur du groupe ", elle "accroche l'homme dans son univers concret » (Ibid. : 29, I03); de l'autre, «l'acrobatie, les exercices d’équilibre, la danse matérialisent dans une large mesure l'effort de soustraction aux chaînes opératoires normales" (Ibid.: IO3). Le rythme renvoie donc autant à la régularisation qu’à la rupture de " chaînes opératoires machinales ", dans leur "durée propre ". Dans les deux cas, il n’est ni répétition ni identité, mais médiation entre deux ordres de grandeur hétérogènes, production d'un certain rapport de mouvements entre milieux.

Ainsi saisie dans sa dynamique la plus élémentaire, comme établissement d'une harmonie entre des rythmes qui saccordent, l'«insertion dans l'existence " a d'emblée pour André Leroi-Gourhan une valeur esthétique. Il étend en effet le champ de l'esthétique, par-delà les créations proprement artistiques, à "l'ensemble du vécu " susceptible de "création rythmique " (Bromberger I988: 158). De cette redéfinition de l'esthétique, témoigne la considération de "l'esthétique physiologique": notre auteur souligne les «implications esthétiques» du comportement nutritif, de l'affectivité physique et de l'équilibre spatial. $\mathrm{Si}$ « la conquête de l'esthétique est aussi importante que celle de l'outil et du langage », c'est que «le fait de ressentir les valeurs esthétiques de la nature est un fait paléontologique" (LeroiGourhan 1965 a : 86; 1982 : I82). Activité proprement humaine, à l'égal de la technicité et du langage, l'esthétique englobe alors le domaine de ce qui prend valeur, en étant apprécié comme "bon ", «beau ", «bien ", " mieux ", etc. ${ }^{7}$ Dès lors, toute valeur est d'ordre esthétique et recouvre une production rythmique, celle d'un milieu relativement unifié. La «sensation d'équilibre "

7 Ce sens de la notion de valeur est celui couramment utilisé par les épistémologues de la biologie (Canguilhem 1988). 
vaut ainsi par elle-même; la "saveur " de l'équilibre rythmique est celle d'une "proximité" conquise entre des corps, des gestes, des formes. Les "jugements physiologiques de valeur ", manifestant la qualité affective d’une convenance intime, saisie "dans toute l'épaisseur de la vie sensitive", témoignent du caractère éminemment créateur des « fonds physiologiques et fonctionnels" sans pour autant que cette "appréciation esthétique des formes" ne conduise vers une «symbolisation figurative». L'esthétique ne commence pas avec la figuration : dès l'ordre physiologique et fonctionnel, "des jugements de valeur sont possibles et conditionnent des normes qui ne sont ni totalement techniques, ni morales, mais esthétiques » (Ibid. : 84-85).

"L'esthétique repose sur la conscience des formes et du mouvement (ou des valeurs et des rythmes) propre à lhomme parce quill est le seul à pouvoir formuler un jugement de valeur " (Ibid. : 95).

«Une part importante de l'esthétique se rattache à l'humanisation de comportements communs à l'homme et aux animaux, comme le sentiment de confort ou dinconfort, le conditionnement visuel, auditif, olfactif, et à lintellectualisation, à travers les symboles, des faits biologiques de cohésion avec le milieu naturel et social [...]. Les références de la sensibilité esthétique, chez l'homme, prennent leurs sources dans la sensibilité viscérale et musculaire profonde, dans la sensibilité dermique, dans les sens olfacto-gustatifs, auditif et visuel, enfin dans limage intellectuelle, reflet symbolique de l'ensemble des tissus de la sensibilité "(Ibid. : 83).

Confort physique et cohésion : l'appréciation esthétique recouvre ainsi une production rythmique. Et ce, de l'esthétique physiologique jusquaux formes les plus élaborées de symbolisation esthétique.

C'est en effet également à partir du champ de la motricité humaine qu'André Leroi-Gourhan saisit la genèse des formes de symbolisation. Si "lintellectualisation progressive des sensations" lui semble avoir ici «dépouillé les formes réelles de leur contenu pour n’en conserver que les signes ", c'est en conservant toujours la même source rythmique : se joue là l'extériorisation du mouvement dans des symboles rythmiques. La figuration est en ce sens une figuration motrice, et le symbole celui d'un "déroulement moteur»; il "ne puise pas ses références dans la mécanique digestive, imperméable à la figuration, mais dans la dynamique musculaire, base commune au comportement affectif et à l'intégration spatiale». Ce que l'homme - comme être agissant- réfléchit dans un réseau de symboles, est avant tout la trame de mouvements «issus de l'extérieur ou de sa propre machine » dans laquelle il est pris. 
«Si l'homme a un merveilleux appareil à transformer les sensations en symboles [...] il n'en reste pas moins quil vit toute l'épaisseur de la vie sensitive " (Ibid. : 95).

« L'art le plus pur plonge toujours dans les profondeurs, il émerge tout juste par la pointe, du socle de chair et d'os sans lequel il ne serait pas " (Ibid. : 88).

«Les manifestations physiologiques trouvent une place prépondérante dans les opérations quotidiennes et figurent à la fois le substrat paléontologique le plus profond et le domaine le plus fréquenté par les sujets vivants » (Ibid. : 92).

De « la satisfaction digestive au bel outil, à la musique dansée, à la danse regardée dans un fauteuil, il y aurait le même phénomène d'extériorisation " (Ibid. : 89) : c'est la rythmicité, la plus concrète comme la plus figurée, qui nourrit et suscite l'appréciation esthétique. Une dimension esthétique est ainsi inhérente à la genèse continuée par l'homme de médiations assurant son insertion dans son milieu de vie: la rythmicité est la matière même de ces médiations. On ne saurait done se méprendre sur la nécessité de «chercher dans la perception et la création de symboles rythmiques une source enfouie dans le monde animal». Il s'agit seulement là de considérer la valeur immédiatement sensible et affective du mouvement: linsertion, à même lintégration motrice, s'élabore de façon de plus en plus médiate jusqu'aux symboles rythmiques, image d'une insertion possible. À travers eux, on ne fait plus, on s'imagine faire : linsertion n'est plus immédiate, elle ne passe plus seulement par une "participation réflexe aux rythmes et une réaction aux variations dans les valeurs", mais par la "perception et la production réfléchie des rythmes et des valeurs", à laquelle aboutit chez l'homme «lintellectualisation progressive des sensations " (1965a : 82).

Ainsi, nous pouvons comprendre la centralité de la question du rythme dans son étude de lart pariétal préhistorique (1965b, 1992), ou du « rythme statuaire 8 (1970), abordant les figurations artistiques à travers leurs "dispositifs rythmiques". De même, nous comprenons que son abord des religions de la préhistoire (200I) - pour citer une autre œuvre contemporaine du Geste et la Parole- se tienne à égale distance des thèses de « l'art pour l'art» et de l'«envoûtement de chasse », pratique magique devant assurer la

\footnotetext{
${ }^{8}$ Comme celle des grottes ornées, l'esthétique des statuettes paléolithiques est rapportée à « une intégration rythmique relativement heureuse ». Le chercheur s'emploie à expliciter les "formules rythmiques» et les mécanismes de cette «suggestion rythmique " sur quoi repose le sentiment d'« équilibre ", de "cohérence», «l'impression d'harmonie » éveillé par ces figurines, donc leur "valeur esthétique»? Si le statisme des figures le conduit ici à « limiter le problème du rythme aux lignes de construction principales et aux proportions », il voit dans le cadrage et les intervalles isométriques les deux principaux «éléments rythmiques de la statuaire primitive »-probablement inexplicites mais essentiels (Leroi-Gourhan 1970 : 661, 672).
} 
possession des animaux figurés (I965b: IOI, II9; I982: I87, 360). Appréhendée à travers son "sommet figuratif ", la religion paléolithique livre avant tout « une certaine image de l'ordre universel» (200I : I52), renvoyant $\mathrm{au}$ «besoin à la fois physique et psychique d'assurer une prise de lindividu et du groupe social sur l'univers, de réaliser linsertion de l'homme, à travers l'appareil symbolique, dans le mouvant et l'aléatoire qui l'entourent" (200I : 8o). De sorte que « le comportement religieux, sur un autre plan, est aussi pratique que le comportement technique, il assure comme celui-ci l'intégration de l'homme dans un monde qui le surpasse et avec lequel il négocie physiquement ou métaphysiquement » (200I : 6).

\section{Leroi-Gourban et Lévi-Strauss: rythmicité et théorie du symbolisme}

Par sa "paléontologie des symboles", attentive aux «fondements corporels des valeurs et des rythmes", aux "reliefs profonds du jugement physiologique", André Leroi-Gourhan développe ainsi une conception pragmatique du symbolisme. Relativement à l'anthropologie sociale, qui poursuit depuis Émile Durkheim et Marcel Mauss une théorie sociologique du symbolique, son approche entretient alors une parenté avec celle de Claude Lévi-Strauss : leur commune critique d'un sociologisme qui fait du collectif la source et la cause des attitudes et des représentations individuelles, les mène vers une genèse symbolique du social. Ce faisant, sobserve un glissement d'une topique de la contrainte à une topique de l'attachement dans l'abord du « social » : le caractère opératoire et performatif du symbole, qu'ils situent tous deux dans l'élément de l'affect, consiste en une mise en ordre du monde, un «enracinement ", ou encore une «insertion" ayant une valence esthétique?.

Mais André Leroi-Gourhan fait en ce sens un pas supplémentaire. Claude Lévi-Strauss tient le symbolisme pour une pure activité de l'« esprit humain ", s'éprouvant lui-même dans le jeu de ses facultés. En lỉnstituant en véritable a priori, il prévient efficacement le réductionnisme ordinaire, qui subordonne les faits symboliques à une « fonction pratique évidente », ou qui

\footnotetext{
${ }^{9}$ C. Lévi-Strauss s'est peu à peu démarqué du modèle linguistique auquel il avait initialement souscrit pour lui préférer le modèle musical, seul à même d'offrir une compréhension de cette action immédiatement sensible qui fait l'efficace et la jouissance des mythes (Hénaff $1991: 240$ ).
} 
les explique par les données du psychisme individuel : "la sociologie ne peut pas expliquer la genèse de la pensée symbolique, elle doit la prendre comme donnée " écrit-il (cf. Hénaff I99I : I79). Mais, du même mouvement, il sinterdit de comprendre l'exigence de "mise en ordre" qui anime le symbolisme -véritable énigme, comme la nature même de l'efficacité symbolique, alors fondue dans celle des faits de culture. Or, pour André Leroi-Gourhan, le symbolisme est bien lui-même justiciable d'une genèse, historique et logique, et sa "paléontologie des symboles" est aussi une compréhension de leur efficience. Que le symbolisme engage la dimension de l'affect et de l'esthétique n'implique pas que la pensée doive se limiter à en décrire la cohérence interne en s'arrêtant au seuil de sa genèse et de son efficience. Cela exige au contraire qu'elle réintègre la dimension de l'affect pour se faire pensée du sensible, pensée sensible ${ }^{10}$.

La "paléontologie des symboles » développée par André Leroi-Gourhan, dans son constant souci d'une continuité entre base et sommet, profondeurs et affleurements, source et pointe, esquisse une telle pensée, dont la rythmicité est l'opérateur même. Si Claude Lévi-Strauss n'affranchit peutêtre pas l'anthropologie des apories du sociologisme et du psychologisme, c'est a contrario faute de conférer un véritable statut conceptuel à l'affect ${ }^{11}$. La vive critique durkheimienne d'une genèse affective du social le garde de tout écart à l'égard des traditionnelles dichotomies du sensible et de lintelligible, du psychique et du social, du physiologique et du social, du corps et de la pensée, etc. ${ }^{12}$ On sait pourtant la place majeure qu'accorde le Durkheim des Formes élémentaires de la vie religieuse aux manifestations affectives dans la description du phénomène rituel. L' efficacité réelle » du rite réside dans la production d'intenses moments d' "effervescence ", qui "dépendent du fait même que le groupe est assemblé, non des raisons spéciales pour lesquelles il est assemblé »; le caractère sérieux et vital des rites tient dans cette seule proximité et ce mouvement des corps assemblés: «leur premier effet est donc de rapprocher les individus, de multiplier entre

\footnotetext{
10 Là réside une condition de toute pensée de la rythmicité et des rythmes (Duflo 1996).

11 Le problème peut être formulé en ces termes : si l'élément du symbolique est celui de l'affect, s'il est une genèse symbolique du social, comment refuser de penser la dimension proprement affective du social?

12 J. Goody a livré une profonde critique des nombreuses dichotomies qui structurent l'œuvre de C. LéviStrauss. Nous ne pouvons qu'y renvoyer le lecteur. Rappelons que C. Lévi-Strauss définit le symbolisme comme une opération intelligible réalisée à même le donné sensible (Goody 1979).
} 
eux les contacts et de les rendre plus intimes. Par cela même, le contenu des consciences change " (Durkheim I990: 552, 497).

Par sa filiation maussienne, André Leroi-Gourhan rejoint bien ce second Durkheim, qui ne rabat pas l'affect sur le psychisme individuel. Sa filiation la plus profonde est toutefois probablement bergsonienne. Manifeste dans sa posture critique à l'égard de tout intellectualisme, comme de tout substantialisme de lindividu ou du social, elle sous-tend son parti pris d'une pensée du vivant humain, comme à la fois pensée de la genèse et pensée de la relation, dans laquelle la catégorie du problème remplace celle du besoin (Douçot 2008). Plus fondamentalement encore, elle éclaire le statut nodal quil accorde à la motricité - et à la rythmicité comme "insertion dans l'existence». La philosophie d'Henri Bergson entend en effet opérer une genèse de l'expérience à partir du mouvement. Si notre présent est fondamentalement "sensoriel et moteur", pris dans les "nécessités de l'action ", si « tout vivant est un centre d'action et tout état de conscience une question posée à l'activité motrice", si donc "nous sommes ce que nous faisons et nous nous créons continuellement nous-mêmes ", le meilleur critère de précision de la pensée consiste pour le philosophe à partir de la corporéité et du mouvement en tant quil se constitue (Bergson I99I : 370, 624, 717, 500).

La façon dont André Leroi-Gourhan situe la notion de «tendance » au cœur de son analyse des techniques (1943: 27, 1945: 396) renvoie précisément à cette expansion du mouvement par laquelle lindividu devient sujet de son milieu. Le caractère problématique de la relation au milieu découle en effet de la tendance au mouvement, confrontée à la matière. De même que la notion est introduite "pour exprimer ce qui se situe en deçà de l'acte technique matérialisé dans les gestes et les instruments" (Ibid.: 326), de même, la recherche d'un contact efficace est au principe de l'« ethnologie de la civilisation industrielle » ou «paléontologie du geste»: «il y a dans la presque totalité des actes techniques la recherche du contact, du toucher " (I945: $384-387$ ). Milieu et techniques déploie cette pragmatique du contact, de l'alliance industrieuse entre la main et le milieu, où le "contact efficace " engage avant tout la possibilité d'une expansion du mouvement lui-même : "amplification de notre être moteur", selon la belle expression de Maurice Merleau-Ponty (I993: 245). Le souci d'efficacité nïmplique ainsi par luimême aucun utilitarisme: il sordonne au renouvellement et à lindétermination de notre puissance d'agir. Le statut de la routine chez 
André Leroi-Gourhan en témoigne: bien qu'elle permette d'agir efficacement sur le milieu, elle "existe pour céder", elle n'est jamais qu’un «fonds précaire» pour une «activité créatrice " (Leroi-Gourhan I945 : 395, 426). La notion de tendance désigne cette dynamique motrice. Prenons l'exemple du rouet, dont linvention marque l'accès au mouvement circulaire continu. Il ne préexiste pas dans le fuseau, ni n’est préformé dans la tendance à tordre du fil: «c'est par une illusion commode que nous prêtons à la tendance l'intention de créer le rouet". La seule tendance permanente est celle de l'activité motrice : "tordre du fil plus vite et plus commodément " (I943 : 252). Et ce désir noccasionne la production d'une série de médiations que par autant d'« actes volontaires de création » qui, procédant du désir de mouvement, en autorisent de nouvelles libérations ${ }^{13}$.

Ce statut de la motricité résume le hiatus séparant l'anthropologie de Claude Lévi-Strauss de celle d'André Leroi-Gourhan: elle autorise au second la conception pragmatique du symbolisme qui manque au premier pour se défaire de laporie d'une opposition entre utilité et gratuité. L'opération de «mise en ordre » a bien une valence pragmatique : seule cette liaison intrinsèque du symbolisme à la motricité et à la rythmicité permet de saisir la genèse et l'efficacité du symbolique. Il est significatif que Claude Lévi-Strauss n'approche cette hypothèse que de façon réductrice: le symbolisme pourrait avoir pour «fonction" de parer à une anxiété de la pensée devant la réalité. Or nous avons vu que la «paléontologie des symboles" développée par André Leroi-Gourhan, loin de tout psychologisme, part au contraire de la formule hominienne; d'emblée ouverte sur la socialité, elle n'est pas un besoin intellectuel à parer, mais une relation au milieu, vitale et problématique, par laquelle l'homme ne cesse de

\footnotetext{
13 Les notions d'invention et d'activité créatrice sont omniprésentes chez A. Leroi-Gourhan (ainsi 1945 : 395401). Contrairement à la critique menée par B. Latour, son ambivalence est patente à l'égard de la notion d'invention technique : souhaitant «éviter tout ce qui peut prendre une couleur de théorie d'évolution technique », A. Leroi-Gourhan insiste, entre le rouet et le fuseau, sur «la place d'une invention, c'est-à-dire d'un acte volontaire de création ». Mais, affirme-t-il simultanément, " on ne connait le nom d'aucun des inventeurs ». Par la notion de "milieu technique", il fait valoir le caractère distribué des schèmes techniques et corrélativement «la pauvreté relative» en formes techniques originales : de ce second point de vue, la vérité est alors à rechercher dans la continuité de l'effort technique et les influences réciproques qui caractérisent ce milieu : «on n'invente le rouet ou on ne l'emprunte que si l'on est en l'état de l'utiliser». Ainsi par exemple, « la roue de voiture est une révolution technique inaccessible d'emblée, c'est un élément dans l'ensemble auquel participent aussi bien le rouleau à faire glisser des pierres que le tour de potier, le rouet, la roue hydraulique et il est peu vraisemblable que sans aucun de ces derniers appareils un char surgisse du plus ingénieux des cerveaux. L'invention du char s'est faite dans un monde déjà bien préparé » (1943 : 40, 253, 103, 320, 140).
} 
se construire comme «homme global» (I982 : I8). La différence est de taille. Elle permet de comprendre qu'il n'y ait "d'autre art qu'utilitaire" sans convoquer le cadre utilitariste d'une orientation en finalité ni un fonctionnalisme soumettant la figuration à un ordre donné ${ }^{14}$. La «signification pragmatique » des symboles est, pour André Leroi-Gourhan, celle de ce "langage émotionnel dont une part des valeurs est d'origine biologique très générale et dont le cadre de symboles est au contraire fortement spécifié" (1965a). C'est sur cette efficacité du symbolisme qu’il nous faut à présent nous pencher pour saisir la conception pragmatique de la socialité qui oppose si fortement la pensée d'André Leroi-Gourhan à l'analyse lévi-straussienne de l'origine symbolique du social : la socialité va s'éprouver dans l'ordre de la motricité -comme celle d'individus en mouvement, en deçà d’un monde achevé, ou figé par le " conditionnement ".

\section{De l'efficacité de l'esthétique sociale: une pensée pragmatique}

L'efficacité du symbolisme s’énonce chez André Leroi-Gourhan sur un mode générique. Vecteurs d'une mise en ordre du monde et d'une "sécurité opératoire ", les symboles participent de plain-pied à linsertion de l'homme «dans l'existence». Si leur genèse nous les a présentés comme limage et l'extériorisation du mouvement, leur efficience est pour André LeroiGourhan d'ordre "pratique» 15 : ils offrent autant d'appuis à l'activité humaine dans son souci intrinsèque de création d'un milieu, d'une "enveloppe rythmique" (1965a : 135). C'est ainsi à la rythmicité du pas, qui préside à la fois à lintégration spatio-temporelle et à l'animation de la vie sociale, qu'André Leroi-Gourhan réfère aussi bien les dispositifs symboliques assurant la domestication du temps et de l'espace que ceux relatifs aux rapports didentification interindividuels, ce vaste espace symbolique de la reconnaissance :

«Le sceptre, symbole de la puissance royale, la crosse, houlette symbolique de l'évêque, la chanson d'amour, l'hymne patriotique, la statue matérialisant le pouvoir des dieux, la

\footnotetext{
${ }^{14}$ A. Leroi-Gourhan se défend d'ailleurs de tout fonctionnalisme (1982:17).

15 Nous préférons pour notre part l'adjectif «pragmatique ( également utilisé comme synonyme par A. LeroiGourhan [1965a : 208]), qui s'oppose mieux à la tendance ordinaire, que A. Leroi-Gourhan ne cesse lui-même de repousser, à référer immédiatement l'adjectif « pratique » à l'ordre « matériel » ou « utilitaire » des « besoins de la survie matérielle» $(2001: 5)$.
} 
fresque remémorant les horreurs de l'enfer répondent à des nécessités pratiques indiscutables. La gratuité n'est pas dans les mobiles mais dans la floraison du langage des formes " (Ibid. : 207).

La valence pragmatique de l'esthétique sociale, cette pointe symbolique de la socialité, innerve alors toute la description des symboles de la société : l'horizon d'une «sécurité opératoire" trace une continuité entre la "domestication du temps et de l'espace" et les "rapports d'identification interindividuels ». Abordons successivement ces deux domaines.

La "domestication du temps et de l'espace " est essentiellement une affaire de rythmes : "les rythmes sont créateurs de l'espace et du temps, du moins pour le sujet» (Ibid. : ${ }_{355}$ ). Elle désigne le passage d’une rythmicité naturelle à une rythmicité «régulièrement conditionnée dans le réseau des symboles calendériques, horaires, métriques ": "À ces rythmes donnés se superpose limage dynamique du rythme que l'homme crée et façonne dans ses gestes et dans ses émissions vocales, puis finalement la trace graphique fixée par la main sur la pierre ou l'os" (Ibid.: 139, I44). Ancrée dans la "perception tout animale du périmètre de sécurité, du refuge clos, ou des rythmes socialisants ", elle se déploie à partir du Paléolithique supérieur dans un dispositif proprement symbolique, substituant à la rythmicité chaotique du monde naturel celle des cadences et des intervalles régularisés. Créant, dans l'espace habité et à partir de lui, "un espace et un temps maîtrisables ", elle est alors pour André Leroi-Gourhan «l'élément principal de la socialisation humaine, limage même de l'insertion sociale » (Ibid. : I4O, I42).

«Vu par les animaux, l'homme apparaîtrait comme obsédé par le temps et l'espace qui dominent ses préoccupations sous toutes les formes de sa pensée depuis l'apparition de la civilisation. Conquête de l'espace, grignotage du temps tissent sa vie pratique, bercent son rêve philosophique, alimentent son rêve spirituel. Son grand jeu depuis des millénaires est celui d'organisateur du temps et de l'espace dans le rythme, le calendrier, l'architecture " (Ibid. : I06-IO7).

C’est ainsi dans l'élément de la motricité que s'éprouve linsertion sociale, donc aussi l'efficacité du symbolisme : «le sujet est inséré socialement dans la mesure où il déroule ses chaînes opératoires sans heurts, au fil des moments normaux de l'existence " (Ibid.: 29). De l'exigence proprement vitale d'une organisation systématique de l'espace habité au réseau rythmique rigoureux propre à un système social et à une centralisation urbaine développée, le souci de la rythmicité apparaît au coeur des préoccupations proprement humaines et des formes de symbolisation. La propriété fondamentale des villes est ainsi de donner, via le géométrisme et la mesure du temps et de 
l'espace, « une vision ordonnée de l'univers ». Dès la pensée cosmologique, la recherche d'une continuité ordonnée entre l'espace urbanisé et le monde qui l'entoure tend vers ce point où, nous dit André Leroi-Gourhan, « la sécurité de l'homme est devenue totale puisque tout est expliqué, saisi, fixé" (Ibid.: I66). C'est en ce sens aussi qu'il établit «le caractère hautement symbolique " de l'art pariétal, attestant au Paléolithique supérieur une "métaphysique véritable» (1982: 156). Les grottes ornées sont de véritables «sanctuaires organisés " pour celui qui étudie, à partir des données tirées de lintégration spatiale (proportions, symétrie, perspective) et de l'intégration temporelle (animation), «l'équilibre régnant entre la majorité des figures", dans leurs "compositions répétées » et leurs "qualités rythmiques »16 (I992: 225, 230, 246).

"Créer une surface artificielle qui isole l'homme comme un cercle magique n'est pas séparable du fait de pouvoir y faire entrer, matériellement ou symboliquement, les éléments maittrisés de l'univers extérieur, et il n’y a pas une grande distance entre l'intégration du grenier, réserve de nourriture, et celle du temple, symbole de lunivers contrôlé $[. .$.$] . Si le tissu de symboles qui recouvre la réalité fonctionnelle des institutions$ humaines offre d'une civilisation à l'autre d'aussi extraordinaires coïncidences, c'est précisément parce quili se moule sur des reliefs profonds [...]. Ce n'est pas par une sorte de raffinement intellectuel gratuit qu'une cité est l'image du monde » (1965a : I68, I83).

Il n'est pas en effet de thème plus constant chez André Leroi-Gourhan que celui de l'« équilibre dynamique entre la sécurité et la liberté », entre les deux faces de "l'assurance matérielle ou métaphysique » et de « la lancée dans une exploration efficace" (Ibid.: I7I). L'« échappée libératrice», «l'activité créatrice ", supposent " le confort d'une parfaite insertion" (Ibid. : I77, 208). Les deux visages du rythme sont bien liés : s'arracher à la rythmicité propre à un milieu, c'est commencer, avec son appui, à organiser la rythmicité d'un autre. À travers le symbolisme, lélaboration d’une image cohérente du monde est alors un trait du comportement humain aussi fondamental que l'activité manuelle ou le langage. Cette exigence de "confort dans l'activité créatrice " fait même la continuité du social, entendue comme convention rythmique, avec ce qu'André Leroi-Gourhan regarde comme le fait le plus fondamental de l'existence biologique : «lintégration dans un milieu dont il

\footnotetext{
16 Une attention toute particulière est portée au pictogramme : «figure ou groupe de figurées coordonnées dans un réseau spatial et évoquant le temps et l'action par la figuration de mouvements significatifs " (1992: 275). Dans le mythogramme, plus largement représenté, les éléments sont en effet simplement juxtaposés, sans référence au temps et à l'espace ; dans l'idéogramme, les figures sont réduites à quelques traits ou à un tracé géométrique.
} 
faut vivre et dans lequel il faut survivre ". Le symbolisme social sous-tend en effet, avec le déploiement de mouvements rythmiquement adéquats, la créativité propre à la motricité et l'activité humaine :

"La sécurité opposée à la liberté, c'est aussi l’opposition entre l'ordre et le chaos qui n’est pas désordre mais promesse d'organisation efficace, c'est l'opposition entre la rythmicité des opérations quotidiennes et l'évasion dans les opérations exceptionnelles [...]. C’est le même cycle qui joint limmobilité au mouvement, la sécurité à la liberté, le confort à lacquisition, le refuge au territoire " (Ibid. : 172 ).

C'est sur le même mode qu'André Leroi-Gourhan envisage les « rapports d'identification interindividuels": les processus de reconnaissance sociale qui s'opèrent essentiellement à travers la parure, les attitudes et le langage. Permettant de situer et d'identifier chacun, autorisant « la prise de contact et lusage approprié des attitudes et du langage ", la parure et le vêtement ont à ses yeux une valeur plus pragmatique que protectrice, au sein même des espèces animales ${ }^{17}$ : l'espèce humaine se singularise par le raffinement intellectuel qui lui permet «d'accumuler les symboles de séduction ou d'effroi ». En ce sens, la parure constitue pour André Leroi-Gourhan « une tendance " (1943: 27). Les attitudes et le langage viennent alors compléter la reconnaissance et organiser le comportement de relation.

C'est cette dimension pragmatique de l'uniforme de gestes, de formules et de traits vestimentaires que chacun endosse en tant quill appartient à un groupe, à une culture déterminée, qui intéresse André Leroi-Gourhan et le prévient de tout sociologisme. La valeur pragmatique des normes sociales et des traits culturels prévaut -d'un point de vue à la fois analytique et génétique- sur leur valeur contraignante : c'est en permettant des contacts effectifs d'une part, en représentant l'unité du groupe de l'autre, qu'ils sont décisifs pour "linsertion" de chacun et la "saveur de la proximité " (1965a : 6I) qui fait la substance même de la «survie ethnique». Inaccessibles aux formes langagières, les harmonies propres à la réalité de l'ethnie ne se prêtent qu’à une " perception identificatrice ${ }^{18}$. Si André Leroi-Gourhan définit bien

17 «Ce qui fait la valeur humaine des insignes et des attitudes est leur lien avec la spéciation ethnique et non leur nature»; « La reconnaissance sociale tient fort près des codes qui permettent aux oiseaux d'établir leurs rapports sur des signes de plumage. Mais déjà la frontière est tracée entre l'espace vécu du blaireau et l'espace construit symboliquement par l'homme, entre la parure du coq de bruyère et l'uniforme symbolique de l'officier supérieur, entre le chant du rossignol et la mélodie sentimentale. Pour l'homme il s'agit de comportements vécus à travers le filtre des images et s'il est nécessaire de sentir qu'ils naissent aux niveaux profonds, il deviendrait inutilement paradoxal de les y maintenir » (1965a : 197, 119).

18 Sur ce statut du langage, cf. aussi «Ethnologie et esthétique» (Leroi-Gourhan 1982). 
le style ethnique comme " la manière propre à une collectivité d'assumer et de marquer les formes, les valeurs et les rythmes ", cette socialité instituée n'est donc jamais appréhendée pour elle-même, mais bien toujours en sa qualité d'appui pragmatique, en son lien intrinsèque à la motricité et à l'activité humaine. La valeur pragmatique des symboles sociaux et des «attitudes collectives très caractéristiques" est donc du même ordre que celle du rassemblement des corps et de l'effervescence durkheimienne : ils autorisent, au ras de l'esthétique physiologique ou fonctionnelle, un sentiment d'«insertion dans l'existence ", et avec elle le déploiement "sans heurts " d’une activité créatrice.

\section{Le social comme "charnière »}

S'étendant de la rythmicité physiologique aux «symboles" de l'esthétique sociale, le «social» revêt donc chez André Leroi-Gourhan le statut d'une "charnière ". La formule est explicite :

"Le social occupe la situation de charnière à un double titre. D’une part les opérations sociales offrent toute la gamme des fréquences de pratique et une stylisation qui va du geste machinal de boutonner son vêtement pour avoir une tenue correcte jusquau cérémonial des réceptions de chefs d'État; d'autre part elles offrent une gradation des niveaux de machinalisation qui s'étend du physiologique dans les attitudes du corps par exemple jusqu'au symbolisme abstrait dans le maniement des chiffres ou du calendrier * (1965a : 93)

Le statut accordé à la rythmicité, au-delà des dichotomies classiques nature/culture, individu/société, physiologique/social, sétaye sur la valeur sociale de l'affect. André Leroi-Gourhan se rapproche ici de Gilbert Simondon, écrivant - dans une même critique du psychologisme et du sociologisme-, quil y a «du collectif quand une émotion se structure » et que la stabilité affective est « un caractère de l'être individuel qui est déjà du social " 19 . L'«insertion affective" de chacun s'appuie ainsi autant sur le « réseau artificiel » qui englobe les jours et les distances en une trame spatiotemporelle, que sur les artifices de la séduction. De même, si la guerre, la prise de position hiérarchique et l'amour suffisent à conditionner «le décor

19 «C'est au niveau des thèmes affectivo-émotifs, mixtes de représentations et d'action, que se constituent les groupements collectifs. Les véhicules de cette communauté affective sont alors les éléments non seulement symboliques mais efficaces de la vie des groupes : régime des sanctions et des récompenses, symboles, arts, objets collectivement valorisés ou dévalorisés " (Simondon 1989 : 100). 
vestimentaire de tous les peuples", c'est que le vêtement participe de linsertion de chacun, plus quil n'est contraint par une appartenance. La socialité ne s'autonomise en ce sens jamais chez André Leroi-Gourhan de la créativité des "fonds physiologiques et fonctionnels". Ce serait risquer de perdre la perspective paléontologique :

«Il pourrait suffire de considérer la figuration comme base de l'esthétique, et d'admettre que, par exemple, si la notion de confort physique est différente au Japon et en Chine, ce n'est pas pour des causes physiologiques, mais parce que les normes sociales et artistiques impriment aux individus des attitudes dans lesquelles laccoutumance se traduit par des sensations de confort. On pourrait de même considérer que l'esthétique du geste de politesse n'a pas pour fondement la cohésion sociale, mais qu'elle est le reflet d'une certaine imagination du comportement éduqué, imagination qui trouve son modèle dans l'art cérémoniel, lindividu jouant son rôle d'homme poli. On perdrait, à procéder ainsi, la perspective paléontologique " (1965a : 84).

La confrontation avec Claude Lévi-Strauss s'opère ici terme à terme. Ce dernier écrit en effet :

"Chaque homme ressent en fonction de la manière dont il est permis ou prescrit de se conduire. Les coutumes sont données comme normes externes, avant d'engendrer des sentiments internes, et ces normes insensibles déterminent les sentiments individuels, ainsi que les circonstances où ils pourront, ou devront, se manifester " (Lévi-Strauss $1962: 105)$.

"La formulation psychologique n'est qu'une traduction, sur le plan du psychisme individuel, d'une structure proprement sociologique » (Lévi-Strauss I950 : XVI).

Si sa réévaluation de la rythmicité conduit André Leroi-Gourhan à penser le social comme une "charnière", c'est bien une autre conceptualisation de la socialité, et de laction elle-même, qui est engagée. Pragmatique, elle se donne en une co-genèse de la socialité et de la motricité humaine.

Par sa réévaluation de la rythmicité, André Leroi-Gourhan reconduit l'anthropologie aux dimensions de l'affect et de la corporéité, avant celles du pouvoir et des normes sociales. Considérant les problèmes classiquement durkheimiens du "groupement des hommes" et de «linsertion dans l'existence ", il les saisit depuis une esthétique du mouvement : loin de référer l'activité humaine aux normes et aux façons d'agir propres à un collectif institué, exerçant une contrainte déterminante sur l'agir individuel, il part de 
son déploiement incarné20 et opère une genèse du social dans la motricité. Si du commun affleure, c'est à partir de la "mémoire motrice » et de la rencontre entre des individus en mouvement. Saisir dans la création et l'équilibre rythmique l'étoffe même de la socialité engage ainsi les théories du « social » à passer d'une topique de la contrainte à une topique de l'attachement. C'est en comptant la relation incarnée de l'homme à son milieu au nombre des «activités relationnelles humaines » que l'anthropologie peut penser la genèse de la socialité21.

Appréhendée depuis la rythmicité, la socialité se présente alors comme une «insertion dans l'existence" qui, de ses formes éruptives aux plus symboliques, trame l'appui pragmatique de toute exploration efficace, de toute activité créatrice. La pensée d'André Leroi-Gourhan ouvrirait-elle alors sur un cercle ? La motricité nourrirait linsertion sociale, laquelle s'offrirait en retour comme son nécessaire appui. Ce n'est pas le cas car c'est au niveau de la motricité que se nouent attachement et création. Cette conceptualisation de la socialité engage alors un retour sur l'activité elle-même. Si les valeurs s'entendent chez André Leroi-Gourhan comme des préférences générales fondées sur l'émotion, celle-ci est toujours émotion de créer. Plutôt que simplement choisies, délibérées, ou même apprises, elles apparaissent comme le produit d'une relation incarnée et créative à un domaine de réalité : "le sens de la valeur est celui de l'auto-constitution du sujet par sa propre action " (Simondon 1989: 255-256). Tout le propos d'André Leroi-Gourhan sordonne à ce souci d’une création rythmique regardée comme le lieu même de la « libération individuelle».

Sa réflexion sur la centralité du rythme dans la genèse des valeurs et des modes d'existence humains ouvre aussi sur une analyse du devenir contemporain des formes de rythmicité. André Leroi-Gourhan fait ici montre d'un certain pessimisme. Il pointe un «danger» : la disparition de toute création rythmique, annihilée par un "conditionnement social pratiquement total " et l'assujettissement des individus à une "grille dans laquelle ils seraient bloqués" (Leroi-Gourhan I965a: 20I). Cette « extériorisation du symbolisme social " (Ibid. : 200) poursuivrait le processus

\footnotetext{
20 Des deux figures de la pratique présentes chez M. Mauss, A. Leroi-Gourhan n'en reprend qu'une (Thévenot 1999).

21 Selon l'expression de G. Simondon, estimant que c'est «l'être comme relation qui est premier et qui doit être pris comme principe » de toute étude de l'homme (Simondon 1989).
} 
par lequel l'homme a peu à peu extériorisé ses facultés dans des organes artificiels, en une série de libérations successives (Ibid.: 5०-52). Quand l'Homo sapiens, ayant fini de s'extérioriser, se trouvera "embarrassé par cet appareil ostéo-musculaire désuet, hérité du Paléolithique ", il sera alors "près de la fin de sa carrière ": " comment ce mammifère désuet, avec les besoins archaïques qui ont été le moteur de toute son ascension, continuera-t-il de pousser son rocher sur la pente sil ne lui reste un jour que limage de sa réalité ?" (Ibid.: 266). Lhomme qui serait radicalement coupé de toute "réalité", réduit à recevoir passivement "sa part de vie collective " et sa "ration émotionnelle ", à satisfaire son exigence d’appartenance à travers les "boîtes magiques" - la "fenêtre de la télévision", les "lèvres du transistor »- qui lui présentent «le jeu de quelques représentants conventionnels ${ }^{22}$, perdrait en effet l'un de ses attributs spécifiques: «le privilège individuel de la création matérielle et symbolique" ( $1965 \mathrm{a}:$ 202-205). Cet appauvrissement corrélatif du symbolisme et de la création rythmique rejoint le lien étroit établi entre le devenir de la motricité humaine et celui des formes de symbolisation ${ }^{23}$.

Mais cette inquiétude ne doit-elle pas se lire plutôt comme un raisonnement par l'absurde? Elle montrerait alors qu'une «vie sociale purement figurée » et venant sans à-coups se substituer à « une vie sociale réelle " n'est qu'une vue de l'esprit. Sauf mutation de l'espèce, lincarnation humaine est là en effet, qui rend aussi inconcevable un symbolisme social coupé de sa source vivifiante qu'une extinction de toute création rythmique «personnelle». La rythmicité connaîtrait plutôt des déplacements. André Leroi-Gourhan prend le parti d'un individu « socialisable à l'infini ", quel que

\footnotetext{
22 L'ironie et l'outrance ne préviennent pas complètement le passéisme porté par la vivacité de son inquiétude : ces hommes «assistent non pas à une cérémonie villageoise mais aux réceptions des grands de ce monde, ils assistent non plus au mariage de la fille du boulanger, mais à celui des princesses; les équipes de football sont les meilleures du continent et ils disposent du meilleur angle de vision. Les neiges du Canada, les sables du désert, les danses papoues, les orchestres du meilleur jazz ruissellent sur eux par les ouvertures de boîtes magiques [...]. La multitude, elle, ne chantera plus aux noces, ne suivra plus la retraite aux flambeaux : dans ses courtes promenades, elle peut déjà éviter le contact direct avec le chant des oiseaux en forçant un peu le ton du transistor». Et d'imaginer dans dix générations les «stages de renaturation dans un parc» où le " créateur de fictions sociales » sera placé pour s'essayer à "retourner un coin de terre ", à « jouer une noce ", à «vendre des choux à d'autres stagiaires sur un petit marché », à " organiser des visites de voisinage », ou pour réapprendre «à confronter les très vieux écrits de Flaubert à la réalité maigrement reconstruite " (1965a : 202203).

23 «L'imagination est la propriété fondamentale de l'intelligence et une société où la propriété de forger des symboles s'affaiblirait perdrait conjointement sa propriété d'agir » (Leroi-Gourhan 1964 : 296).
} 
soit le caractère artificiel ou technicisé de son environnement : «il vaut mieux jouer sur l'homme» (Ibid. : 267).

La sociologie et l'anthropologie du travail montrent l'actualité de cette interrogation. Confrontées à des activités de plus en plus équipées en "technologies intellectuelles", loin des figures classiques du travail mécanique et du métier artisanal (Pillon \& Vatin 2007, Bidet 2006) ou de la "motricité industrielle" (Leroi-Gourhan 1965a: 59), elles ré-ouvrent aujourd'hui la question de la technicité, après celle du langage ${ }^{24}$. Les apports de la tradition française d'anthropologie technique ne sont pas ici sans parenté avec ceux de la tradition pragmatiste américaine. Si les liens entre la philosophie pragmatiste et l'anthropologie culturelle ont connu un éclaircissement récent, ceux qu'elle entretient avec l'anthropologie technique restent à explorer.

A. B.

\section{Références}

Bergson, Henri

199I Euvres. Paris : Édition du Centenaire-PUF.

Bidet, Alexandra

200I «Le travail et l'économique. Pour un regard anthropologique»,

Sociologie du travail (43), 2.

2002 «Au fil des dérangements téléphoniques. Analyse d'une interaction technique ", Réseaux, II5.

2007 "Le travail entre corps et technique. Du labor à l'agir créatif ", Communications 8I : Corps \& techniques.

Bidet, Alexandra (ed.), et al.

2006 Sociologie du travail et activité. Toulouse : Octarès.

Breviglieri, Marc

1993 «Objet et geste technique chez André Leroi-Gourhan ", Document de travail, GSPM.

Bromberger, Claude

I988 "André Leroi-Gourhan et l'esthétique ", in André Leroi-Gourhan ou les Voies de l'Homme. Actes du colloque CNRS. Paris : Albin Michel : 157-I67.

${ }^{24}$ Nous faisons ici référence aux travaux menés depuis plus d'une quinzaine d'années par le réseau « Langage et travail ». Sur la question de la technicité, voir notamment l'ouvrage collectif (Bidet 2006 ; et 2007). 
Canguilhem, Georges

I988 Le Normal et le pathologique. Paris : Presses Universitaires de France ("Quadrige»).

Clot, Yves

I995 Le Travail sans l'homme? Pour une psychologie des milieux de travail et de vie. Paris : La Découverte.

Dodier, Nicolas

1995 Les Hommes et les machines. La conscience collective dans les sociétés technicisées. Paris : Métailié.

Douçot, Julien

2008 "Le vivant en activité. Besoin, problème et créativité chez Henri Bergson ", Archives de philosophie 7I(2).

Duflo, Colas

1996 «Lhumanité du rythme », in P. Sauvanet \& J.-J. Wunenburger (eds), Rythmes et philosophie. Paris : Kimé.

Durkheim, Émile

I990 Les Formes élémentaires de la vie religieuse. Paris : Presses

Universitaires de France.

Goody, Jack

I979 La Raison graphique. La domestication de la pensée sauvage. Paris : Minuit.

Hénaff, Marcel

I99I Claude Lévi-Strauss et lanthropologie structurale. Paris : Belfond.

Karsenti, Bruno

1998 "Techniques du corps et normes sociales : de Mauss à Leroi-

Gourhan ", Intellectica 26-27: 227-240.

Karsenti, Bruno \& Louis Quéré (eds.)

2004 La croyance et l'enquête. Aux sources du pragmatisme. Paris :

EHESS (Raisons pratiques $\mathrm{n}^{\circ}{ }^{\circ}$ ).

Kaufmann, Jean-Claude

1997 Le Cour à louvrage. Théorie de l’action ménagère. Paris : Nathan.

$200 \mathrm{I}$ Ego. Pour une sociologie de lindividu. Paris : Nathan.

Latour, Bruno

1991 Nous n’avons jamais été modernes. Essai d’anthropologie symétrique.

Paris : La Découverte.

Leroi-Gourhan, André

1943 Evolution et techniques, I. L'Homme et la matière. Paris : Albin

I945 Évolution et techniques, II. Milieu et techniques. Paris : Albin

1964 Le Geste et la Parole, I. Technique et langage. Paris : Albin Michel. 
I965a Le Geste et la Parole, II. La Mémoire et les rythmes. Paris : Albin Michel.

I965b Préhistoire de lart occidental. Paris : Mazenod.

I970 "Observations technologiques sur le rythme statuaire ", in Échanges et communications, mélanges offerts à Claude Lévi-Strauss. La Haye : Mouton : 658-676.

I982 Les Racines du monde. Entretiens. Paris : Belfond.

I983 Le Fil du temps. Ethnologie et préhistoire. Paris : Fayard.

I992 L’Art pariétal. Langage de la préhistoire. Grenoble : Jérôme Million.

200I Les Religions de la préhistoire. Paris : Presses Universitaires de France ("Quadrige") (Ière édition I964).

Lévi-Strauss, Claude

I950 "Introduction à l'œuvre de Marcel Mauss ", in Marcel Mauss, Sociologie et anthropologie. Paris : PUF («Quadrige») : IX-LII.

I962 Le Totémisme aujourd'hui. Paris : Presses Universitaires de France.

I973 Anthropologie structurale. Paris : Plon.

Mauss, Marcel

I966 "Les techniques du corps », in Sociologie et Anthropologie. Paris :

Presses Universitaires de France.

Merleau-Ponty, Maurice

I993 Phénoménologie de la perception. Paris : Gallimard (Ière édition 1945).

Pillon, Thierry \& François Vatin

2007 Traité de sociologie du travail. Toulouse : Octarès (Ière éd. 2003).

Sauvanet, Pierre \& Jean-Jacques Wunenburger (eds.)

I996 Rythmes et philosophie. Paris : Kimé.

Schwartz, Yves

I988 Expériences et connaissance du travail. Paris : Messidor.

$200 \mathrm{I}$ "Théories de l'action ou Rencontres de l'activité ", in J.-M. Baudouin \& J. Friedrich (eds), Théories de laction et éducation. Bruxelles : De Boeck : 67-9I.

Simondon, Georges

${ }_{1958} 8$ Du mode d'existence des objets techniques. Paris : Aubier.

I989 Lindividuation psychique et collective. Paris : Aubier Montaigne.

Thévenot, Laurent

I994 "Le régime de familiarité. Des choses en personne ", Genèses I7.

I999 "L'action comme engagement », in J.-M. Barbier (ed.), L'Analyse de la singularité de laction. Paris : Presses Universitaires de France : 213238.

Tinland, François

I977 La Différence anthropologique. Essais sur les rapports de la nature et de l'artifice. Paris : Aubier Montaigne. 